\title{
Epigallocatechin gallate inhibits angiotensin II- induced endothelial barrier dysfunction via inhibition of the p38 MAPK/HSP27 pathway
}

\author{
Dan YANG ${ }^{1}$, Jing LIU1 ${ }^{1}$, Cui TIAN ${ }^{1}$, Yong ZENG ${ }^{2}$, Yue-hong ZHENG ${ }^{3}$, Quan FANG ${ }^{2}$, Hui-hua $\mathrm{LI}^{1,}$ * \\ ${ }^{1}$ Department of Pathology, School of Basic Medical Sciences, Capital Medical University, Beijing 100069; ${ }^{2}$ Department of Cardiology; \\ and ${ }^{3}$ Department of Vascular Surgery, Peking Union Medical Hospital, Beijing 100730, China
}

\begin{abstract}
Aim: To investigate the effect of epigallocatechin gallate (EGCG) on angiotensin II (Ang II)-induced stress fiber formation and hyperpermeability in endothelial cells.

Methods: Human umbilical vein endothelial cells (HUVECs) were treated with Ang II in the absence or presence of EGCG or mitogenactivated protein kinases (MAPKs) inhibitors. The resulting stress fibers were stained with rhodamine-phalloidin and examined using confocal microscopy. The permeability of the endothelium was tested with fluorescein-isothiocyanate labeled bovine serum albumin (FITC-BSA), and the phosphorylation levels of several proteins were determined using Western blot analysis.

Results: Ang II (1-100 nmol/L) treatment markedly provoked stress fiber formation and hyperpermeability in HUVECs in a time- and dose-dependent manner. These effects were abolished by pretreatment with the p38 MAPK inhibitor SB203580 $10 \mu$ mol/L, indicating that the Ang II-induced endothelial barrier dysfunction was via activation of the p38 MAPK/HSP27 pathway. Furthermore, treatment with EGCG (5-25) $\mu \mathrm{mol} / \mathrm{L}$ inhibited Ang Il-induced activation of the p38 MAPK/HSP27 pathway, thereby reducing endothelial stress fiber formation and hyperpermeability.

Conclusion: Our data demonstrate that EGCG inhibits Ang II-induced endothelial stress fiber formation and hyperpermeability via inactivation of p38 MAPK/HSP27 pathway, and suggest that EGCG may protect against endothelial barrier dysfunction and injury.
\end{abstract}

Keywords: angiotensin II; epigallocatechin gallate; endothelial barrier function; mitogen-activated protein kinases; heat-shock protein 27

Acta Pharmacologica Sinica (2010) 31: 1401-1406; doi: 10.1038/aps.2010.75; published online 19 Jul 2010

\section{Introduction}

The vascular endothelium acts as a barrier and plays an important role in maintaining the integrity of the endovascular surface. Damage to the endothelial barrier results in pathological vascular hyperpermeability, which is associated with changes in the filamentous actin (F-actin) cytoskeleton ${ }^{[1]}$. It has been previously reported that actin cytoskeleton reorganization, such as disruption of actin filaments and the formation of stress fibers leads to cell contraction and paracellular gap formation, thereby enhancing endothelial permeability. A multitude of growth factors and proinflammatory cytokines, such as the transforming growth factor-beta (TGF- $\beta$ ), thrombin, and tumor necrosis factor-alpha (TNF- $\alpha$ ) can trigger downstream signaling pathways that initiate reorganization of the actin

\footnotetext{
* To whom correspondence should be addressed. E-mail hhli1935@yahoo.cn

Received 2010-02-25 Accepted 2010-05-24
}

cytoskeleton and increase endothelial permeability ${ }^{[2,3]}$.

Angiotensin II (Ang II) is a prominent vasoconstrictor of the renin-angiotensin system that controls cardiovascular homeostasis and regulates cell growth and inflammation ${ }^{[4]}$. Ang II has multiple effects on endothelial cells, such as production of reactive oxygen species (ROS), apoptotic signaling pathway activation, and thrombosis ${ }^{[5]}$. Furthermore, Ang II increases vascular permeability indirectly via pressure-induced mechanical injury, increases production of prostaglandins, and increases production of vascular endothelial growth factors $(\mathrm{VEGF})^{[6]}$. In addition, Ang II can activate mitogen-activated protein kinases (MAPKs), including extracellular signalregulated kinase1/2 (ERK1/2), c-Jun $\mathrm{NH}_{2}$-terminal kinase 1/2 (JNK1/2), and p38 MAPK $^{[7]}$. A recent study demonstrated that Ang II induces actin cytoskeletal remodeling in human umbilical vein endothelial cells (HUVECs) and bovine aortic endothelial cells (BAECs) ${ }^{[8]}$. Although several studies have shown that the effect of Ang II on actin cytoskeleton is medi- 
ated by Rac $1^{[9]}$ and Rho kinase ${ }^{[10]}$, the mechanisms underlying Ang II-induced endothelial stress fiber formation and hyperpermeability are not well-known.

Epigallocatechin gallate (EGCG), the major catechin that is derived from green tea, protects the cardiovascular system through its anti-oxidative, anti-inflammatory, and antithrombogenic effects ${ }^{[11]}$. It has been suggested that EGCG alleviates Ang II-induced vascular smooth muscle cell (VSMC) hypertrophy by inhibiting JNK1/2 ${ }^{[12]}$. Furthermore, EGCG maintained endothelial barrier function and insulin sensitivity by activating the phosphatidylinositol 3-kinase (PI3K)/AKT/ eNOS pathway ${ }^{[13-15]}$. However, the effect of EGCG on Ang II-induced endothelial stress fiber formation and hyperpermeability remains unclear. In the present study, we determined the role of EGCG in Ang II-induced endothelial barrier dysfunction.

\section{Materials and methods Chemical reagents}

Ang II was obtained from Sigma-Aldrich. Endothelial cell medium (ECM), endothelial cell growth supplement (ECGS), fetal bovine serum (FBS), and penicillin/streptomycin solutions $(\mathrm{P} / \mathrm{S})$ were purchased from Sciencell. Anti-ERK1/2, anti-phospho-ERK1/2, anti-p38 MAPK, anti-phospho-p38 MAPK, anti-JNK1/2, anti-phospho-JNK1/2, anti-HSP27, antiphospho-HSP27, and anti-beta-actin ( $\beta$-actin) primary antibodies, as well as horseradish peroxidase (HRP)-conjugated secondary antibodies were purchased from Cell Signaling Technology. SP600125 and SB203580 were purchased from Calbiochem. EGCG was purchased from Promega, dissolved in sterile water at a stock concentration of $50 \mathrm{mmol} / \mathrm{L}$, and stored at $4{ }^{\circ} \mathrm{C}$.

\section{Cell culture}

HUVECs (Sciencell) were cultured in ECM that was supplemented with $5 \%$ FBS, $1 \%$ ECGS, and $1 \% \mathrm{P} / \mathrm{S}$. The cells were maintained at $37{ }^{\circ} \mathrm{C}$ with $5 \% \mathrm{CO}_{2}$ in a humidified atmosphere as previously described ${ }^{[16]}$. HUVECs were harvested between passages 4 and 6 and were subsequently used for the experiments. Before the indicated treatments, cellular growth was halted by serum starvation. The growth medium was removed and replaced with serum-free medium $12 \mathrm{~h}$ before experimental treatment.

\section{Western blot analysis}

The HUVECs were lysed with extraction buffer $(50 \mathrm{mmol} / \mathrm{L}$ Tris-HCl pH 7.5, 0.5\% Triton X-100, 0.5\% NP40, $150 \mathrm{mmol} / \mathrm{L}$ $\mathrm{NaCl}, 2 \mathrm{mmol} / \mathrm{L}$ EDTA, $1 \mathrm{mmol} / \mathrm{L} \mathrm{NaF}, 1 \mathrm{mmol} / \mathrm{L}$ PMSF, 1 $\mathrm{mmol} / \mathrm{L} \mathrm{Na}_{3} \mathrm{VO}_{4}$, and $10 \%$ protease inhibitors). The extracts were separated by SDS-PAGE on a 10\% gel and were transferred to a nitrocellulose membrane. The membranes were soaked in blocking buffer ( $5 \%$ skim milk), incubated with primary antibodies overnight, then incubated with an HRP-conjugated secondary antibody for $1 \mathrm{~h}$. The blots were developed with a chemiluminescent system, and the relative intensities of the protein bands were determined. To quantify the phospho- specific proteins, we normalized the signal to the amount of total target protein and $\beta$-actin, as described previously ${ }^{[17]}$.

\section{F-actin staining}

The HUVECs were cultured, fixed with paraformaldehyde, and permeabilized for $5 \mathrm{~min}$ with $0.1 \%$ Triton X-100. The cells were subsequently incubated with blocking buffer (1\% BSA) for $30 \mathrm{~min}$ and were stained with rhodamine-phalloidin as previously described ${ }^{[16]}$. The nuclei were counterstained with the DNA-binding dye 2-(4-amidinophenyl)-6-indolecarbamidinedihydrochloride (DAPI). The stained HUVECs were visualized under a confocal microscope (TCS SP2 laser-scanning spectral confocal system; Leica Microsystems, Germany) using a $40 / 1.25$ objective.

\section{Permeability assay}

The HUVECs were cultured in 24-well plates with transwell inserts $(0.4 \mu \mathrm{m}$, Costar). Fluorescein-isothiocyanate labeled bovine serum albumin (FITC-BSA, $10 \mathrm{mg} / \mathrm{mL}$, Sigma) was added to the cells in the upper compartment. The cells were subsequently treated with Ang II in the absence or presence of EGCG or MAPK inhibitors, which were added to both the upper and lower compartments. Cell culture medium was removed from the lower compartment at various time points. The concentration of FITC-BSA was quantified with a fluorescence spectrofluorophotometer as described previously ${ }^{[18]}$. The BSA flux was calculated as a ratio between the fluorescence intensity in the lower compartment and the upper compartment. The data are expressed as a percentage of the control.

\section{Statistical analysis}

Each experiment was performed at least in triplicate. The data are presented as the mean $\pm \mathrm{SD}$. Statistical comparisons were made with the Student's $t$-test, and significance was defined as $P<0.05$.

\section{Results}

Ang II induces endothelial stress fiber formation and hyperpermeability

To determine the effect of Ang II on endothelial cells, HUVECs were treated with Ang II at a concentration of 0, 1, 10, or 100 $\mathrm{nmol} / \mathrm{L}$ for $60 \mathrm{~min}$, or with $100 \mathrm{nmol} / \mathrm{L}$ for $0,5,15,30$, and $60 \mathrm{~min}$. The formation of stress fibers was determined by confocal microscopy, and the endothelial permeability was assessed with the FITC-BSA assay described in the methods. As shown in Figure 1, F-actin was mostly present in cortical structures and was evenly distributed in the control group $(0$ min). In contrast, treatment with Ang II markedly induced the formation of stress fibers and endothelial hyperpermeability in a dose- and time-dependent manner, indicating that Ang II may stimulate endothelial barrier dysfunction.

Ang II-induced endothelial barrier dysfunction is dependent upon the p38 MAPK/HSP27 pathway

To elucidate the mechanisms by which Ang II regulates endothelial barrier dysfunction, we examined the levels of 
A

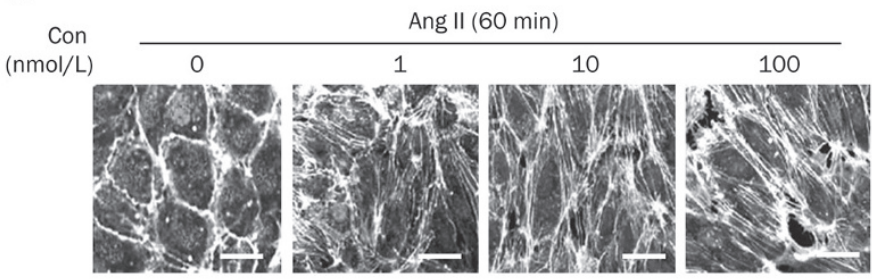

C

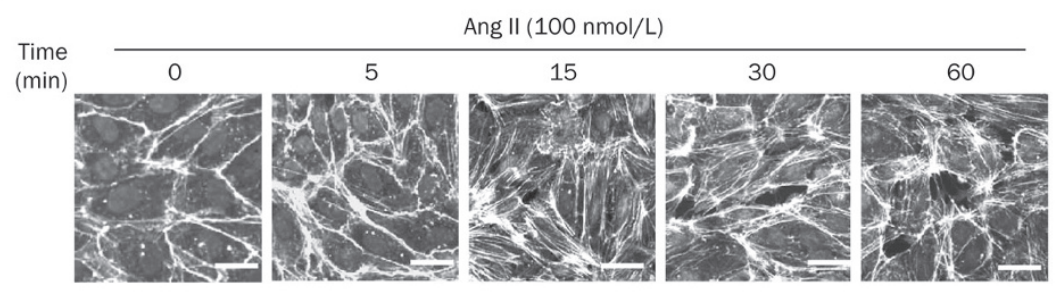

B

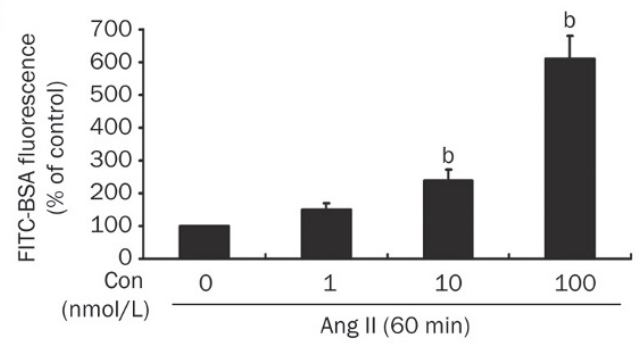

D

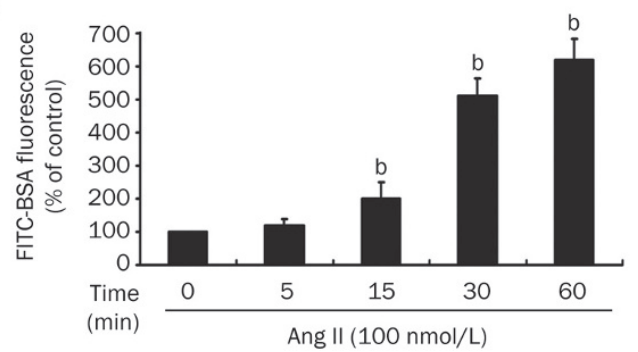

Figure 1. Ang II induces endothelial stress fiber formation and hyperpermeability. (A) HUVECs were treated with Ang II at a concentration of 0 , 1 , 10, or $100 \mathrm{nmol} / \mathrm{L}$ for $60 \mathrm{~min}$. The stress fibers were stained with rhodamine-phalloidin and examined by confocal microscopy $(n=3$, Bar= $20 \mu \mathrm{m})$. (B) HUVECs were treated as in A and the endothelial permeability was determined with a FITC-BSA assay ( $n=4$, mean \pm SD). ${ }^{b} P<0.05 \mathrm{vs} 0 \mathrm{nmol} / \mathrm{L}$ (control). (C) HUVECs were treated with $100 \mathrm{nmol} / \mathrm{L}$ of Ang II for 0,5, 15, 30, and $60 \mathrm{~min}$. The stress fibers were stained and examined as in A ( $n=3$, Bar= 20 $\mu \mathrm{m}$ ). (D) HUVECs were treated as in $\mathrm{C}$, and the endothelial permeability was determined as in $\mathrm{B} .{ }^{\mathrm{b}} \mathrm{P}<0.05$ vs 0 min (control).

p38 MAPK, JNK1/2, ERK1/2, and HSP27 phosphorylation in HUVECs that were treated with $100 \mathrm{nmol} / \mathrm{L}$ of Ang II for various amounts of time. As shown in Figure 2A, Ang II treatment caused an increase in the phosphorylation of p38 MAPK and HSP27, which reached a peak at 15 min and declined to near basal levels at $60 \mathrm{~min}$. Consistent with the pattern of p38 MAPK and HSP27 phosphorylation, JNK1/2 phosphorylation was increased with Ang II treatment and peaked at 15 $\mathrm{min}$, followed by a slow decline after $60 \mathrm{~min}$ (data not shown). In contrast, ERK1/2 phosphorylation was not significantly
A

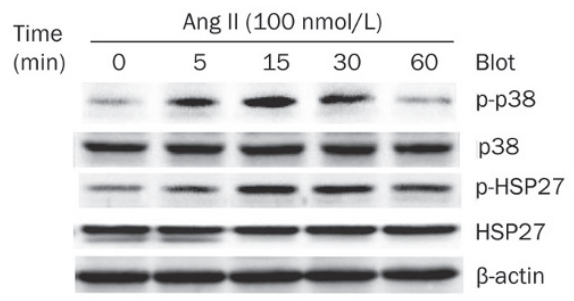

C

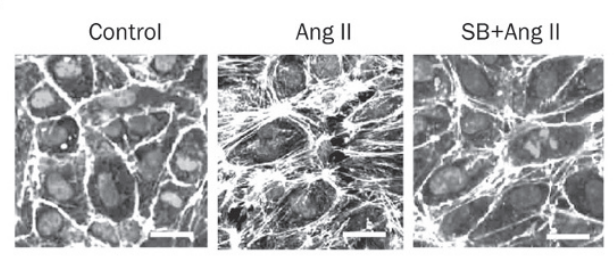

B

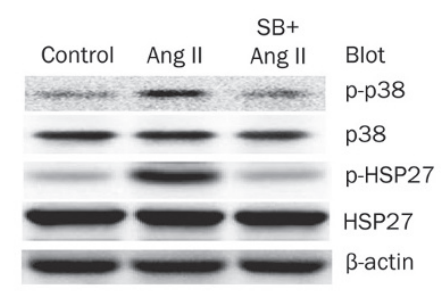

D

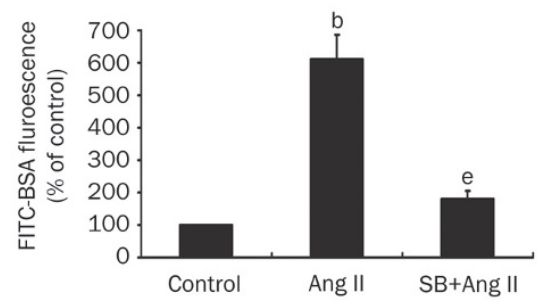

Figure 2. Ang II induces endothelial barrier dysfunction via the p38 MAPK/HSP27 pathway. (A) Ang II induces activation of the p38 MAPK/HSP27 pathway. HUVECs were treated with $100 \mathrm{nmol} / \mathrm{L}$ of Ang II for various periods of time. Phosphorylation of p38 MAPK and HSP27 was detected with a Western blot, and a representative blot is shown $(n=3)$. (B) The effect of SB203580 on phosphorylation of p38 MAPK and HSP27. HUVECs were pretreated with SB203580 (10 $\mu \mathrm{mol} / \mathrm{L})$ for $30 \mathrm{~min}$ before a $15 \mathrm{~min}$ incubation with $100 \mathrm{nmol} / \mathrm{L}$ of Ang II. p38 MAPK and HSP27 phosphorylation was detected as in A, and a representative blot is shown ( $n=3$ ). (C) SB203580 inhibits Ang Il-induced stress fiber formation. HUVECs were pretreated as in $B$ and the stress fibers were stained and examined as in Figure $1 \mathrm{~A}(n=3$, Bar=20 $\mu \mathrm{m})$. (D) SB203580 inhibits Ang II-induced endothelial hyperpermeability. HUVECs were pretreated with SB203580 (10 $\mu \mathrm{mol} / \mathrm{L})$ for $30 \mathrm{~min}$ before a $60 \mathrm{~min}$ incubation with $100 \mathrm{nmol} / \mathrm{L}$ of Ang II. The endothelial permeability was tested as in Figure 1B. $n=4$. Mean \pm SD. ${ }^{\mathrm{b}} P<0.05$ vs control. ${ }^{\mathrm{e}} P<0.05$ vs Ang II-treated cells. 
upregulated by Ang II treatment during the 5-60 min timeframe (data not shown).

To further assess which signaling pathway contributes to Ang II-induced endothelial stress fiber formation and hyperpermeability, specific MAPK inhibitors were tested. Since Ang II efficiently stimulates phosphorylation of p38 MAPK and JNK1/2, the cells were pretreated with the p38 MAPK inhibitor SB203580 (SB, $10 \mu \mathrm{mol} / \mathrm{L})$ or the JNK1/2-specific inhibitor SP600125 (SP, $20 \mu \mathrm{mol} / \mathrm{L}$ ) for $30 \mathrm{~min}$ prior to incubation with $100 \mathrm{nmol} / \mathrm{L}$ of Ang II. As shown in Figure 2B, SB203580 significantly inhibited Ang II-induced phosphorylation of p38 MAPK and HSP27. Although the JNK1/2 inhibitor SP600125 blocked JNK1/2 and c-Jun phosphorylation, it did not decrease HSP27 phosphorylation (data not shown). Furthermore, SB203580 decreased the Ang II-induced formation of stress fibers and endothelial hyperpermeability in HUVECs (Figure 2C, 2D), whereas SP600125 had no effect (data not shown). Collectively, these data indicate that the p38 MAPK/HSP27 pathway plays a critical role in Ang II-induced endothelial barrier dysfunction.

EGCG inhibits Ang II-induced endothelial barrier dysfunction via inhibition of the p38 MAPK/HSP27 pathway

EGCG, which is the major catechin derived from green tea, is associated with a reduced risk of cardiovascular disease ${ }^{[11]}$. Thus, we wanted to determine if EGCG affects Ang II-induced endothelial stress fiber formation and hyperpermeability. The HUVECs were pretreated with 0 to $25 \mu \mathrm{mol} / \mathrm{L}$ of EGCG for $30 \mathrm{~min}$ and subsequently stimulated with $100 \mathrm{nmol} / \mathrm{L}$ of Ang II. As shown in Figure 3A and 3B, EGCG attenuated Ang IIinduced endothelial stress fiber formation and hyperpermeability in a dose-dependent manner, and there was complete inhibition with $25 \mu \mathrm{mol} / \mathrm{L}$ of Ang II. These results demonstrate that EGCG protects against Ang II-induced endothelial barrier dysfunction.

Since activation of the p38 MAPK/HSP27 pathway is required for Ang II-induced endothelial stress fiber formation and hyperpermeability (Figure 2), we next investigated whether EGCG attenuates the Ang II-induced endothelial response via inhibition of the p38 MAPK/HSP27 pathway. HUVECs were pretreated with 0 to $25 \mu \mathrm{mol} / \mathrm{L}$ of EGCG for $30 \mathrm{~min}$ and subsequently stimulated with $100 \mathrm{nmol} / \mathrm{L}$ of Ang II for 15 min. As shown in Figure 4A and 4B, Ang II significantly induced phosphorylation of p38 MAPK and HSP27. This effect was inhibited by EGCG in a dose-dependent manner; at a concentration of $25 \mu \mathrm{mol} / \mathrm{L}$, EGCG completely abolished Ang II activation of the p38 MAPK/HSP27 pathway (Figure 4). Collectively, these results demonstrate that EGCG
A
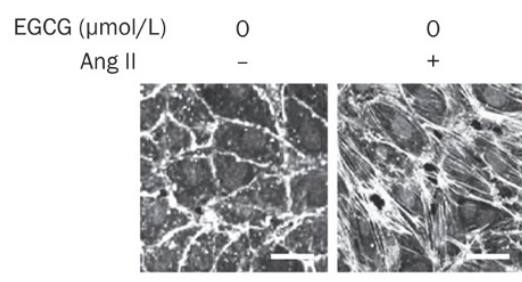

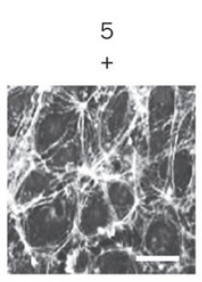

10

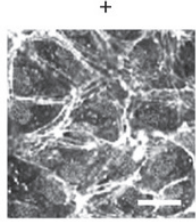

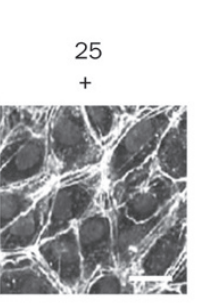

B

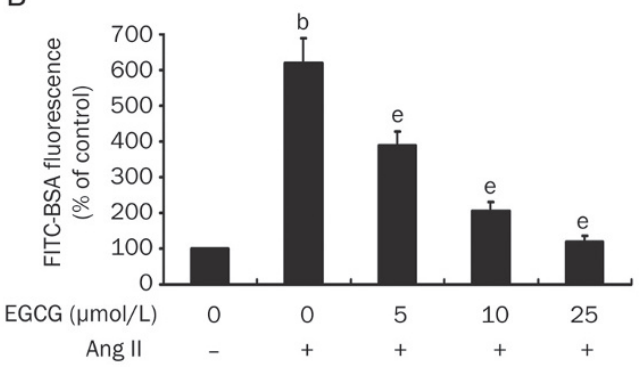

Figure 3. EGCG suppresses Ang II-induced endothelial stress fiber formation and hyperpermeability. (A) The dose-dependent effect of EGCG on Ang IIinduced stress fiber formation. HUVECs were pretreated with 0 to $25 \mu \mathrm{mol} / \mathrm{L}$ of EGCG for $30 \mathrm{~min}$ before a $15 \mathrm{~min}$ incubation with $100 \mathrm{nmol} / \mathrm{L}$ of Ang II. The stress fibers were stained and examined as in Figure $1 \mathrm{~A}(n=3$, Bar=20 $\mu \mathrm{m})$. (B) The dose-dependent effect of EGCG on Ang II-induced endothelial hyperpermeability. HUVECs were pretreated with EGCG as in A prior to a $60 \mathrm{~min}$ incubation with $100 \mathrm{nmol} / \mathrm{L}$ of Ang II. The endothelial permeability was tested as in Figure 1B. $n=4$. Mean \pm SD. ${ }^{b} P<0.05$ vs untreated cells (control). ${ }^{e} P<0.05$ vs Ang II-treated cells.
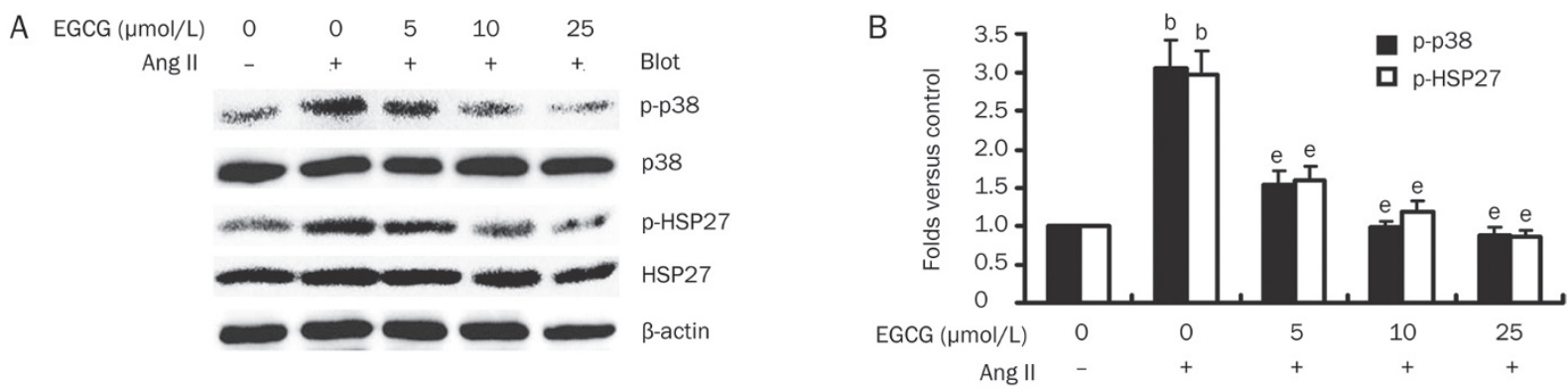

Figure 4. EGCG suppresses Ang II-induced activation of the p38 MAPK/HSP27 pathway. (A) The dose-dependent effect of EGCG on Ang II-induced phosphorylation of p38 MAPK and HSP27. HUVECs were treated as in Figure 3A, and p38 MAPK and HSP27 phosphorylation was detected with a Western blot; a representative blot is shown. (B) The intensity of the phosphorylated p38 MAPK and HSP27 signal is expressed as the fold increase above the control group. $n=3$. Mean \pm SD. ${ }^{\mathrm{b}} P<0.05$ vs untreated cells (control). ${ }^{\mathrm{e}} P<0.05$ vs Ang II-treated cells. 
suppresses Ang II-induced endothelial barrier dysfunction through inhibition of the p38 MAPK/HSP27 pathway.

\section{Discussion}

In this report, we demonstrate that Ang II stimulation drastically increased endothelial stress fiber formation and hyperpermeability. These effects were predominantly mediated via activation of the p38 MAPK/HSP27 pathway. Additionally, EGCG treatment inhibited the p38 MAPK and HSP27 pathway and prevented Ang II-induced endothelial barrier dysfunction. Thus, EGCG may protect against endothelial injury.

The MAPKs, including p38 MAPK, JNK1/2, and ERK1/2 play a role in regulating endothelial permeability and barrier dysfunction during various pathological insults ${ }^{[2,19-24]}$. p38 MAPK is known to regulate a variety of cellular processes, including inflammation, apoptosis, cell migration, and contraction ${ }^{[2]}$. It has been shown that pertussis toxin and TNF- $\alpha$ induce p38 MAPK activation in the endothelium, thereby leading to formation of stress fibers and an increase in endothelial permeability ${ }^{[20,23,24]}$. However, there is little information about the role of MAPKs in Ang II-induced endothelial stress fiber formation and hyperpermeability. In the present study, we have shown that Ang II significantly induces formation of stress fibers and endothelial hyperpermeability in HUVECs in a dose- and time-dependent manner (Figure 1). In addition, treatment of HUVECs with Ang II resulted in a significant increase in phosphorylation of p38 MAPK (Figure 2) and JNK1/2 (data not shown); however, no significant changes were observed in the level of ERK1/2 phosphorylation (data not shown). Interestingly, specific inhibition of p38 MAPK with SB203580 attenuated Ang II-induced endothelial stress fiber formation and hyperpermeability (Figure 2), whereas a specific JNK1/2 inhibitor had no effect (data not shown). These data suggest that activation of p38 MAPK is required for Ang II-induced endothelial barrier dysfunction.

HSP27, an actin-binding protein, is closely associated with the regulation of F-actin assembly ${ }^{[25]}$ and is a known central downstream target of $\mathrm{p} 38 \mathrm{MAPK}^{[26]}$. The activation of p38 MAPK results in activation of MAPK activated protein kinase-2 (MAPKAPK-2), which subsequently phosphorylates HSP27, leads to reorganization of the actin cytoskeleton, and ultimately results in the formation of stress fibers ${ }^{[27]}$. HSP27 is known to play a critical role in actin polymerization in response to TNF- $\alpha$ and various types of stress, including oxidative stress ${ }^{[23,28-30]}$. Similar to the previously published data, our data here show that Ang II markedly induces phosphorylation of HSP27, leading to endothelial stress fiber formation and hyperpermeability. Collectively, these results demonstrate that Ang II induces endothelial barrier dysfunction via activation of the p38 MAPK/HSP27 pathway.

Several studies suggest that EGCG may improve endothelial barrier function through induction of eNOS and NO, which is mediated through the PI3K/AKT pathway ${ }^{[13,15,31]}$. Furthermore, as a potential anti-inflammatory agent, EGCG can prevent the interferon gamma (IFNY)-induced increase in epithelial permeability, which is a STAT1- and MAPK-independent event ${ }^{[32]}$. In addition, EGCG effectively alleviates ritonavir (a HIV protease inhibitor)-induced endothelial hyperpermeability, which is related to the ERK1/2 pathway ${ }^{[33]}$. However, little is known about the effect of EGCG treatment on Ang IIinduced endothelial barrier dysfunction and the related intracellular signaling pathways. In this study, we demonstrate that EGCG inhibited Ang II-induced endothelial stress fiber formation and hyperpermeability via inactivation of the p38 MAPK/HSP27 pathway. Interestingly, a recent study demonstrated that EGCG suppresses phosphorylation of HSP27 when stimulated by sphingosine 1-phosphate via attenuation of the PI3K/AKT pathway; however, EGCG does not impact phosphorylation of the p38 MAPK pathway in osteoblasts ${ }^{[34]}$. Taken together, these results suggest that the effects of EGCG may depend on physiopathological agents and the cells/tissues upon which it acts.

In conclusion, our novel findings provide evidence that Ang II induces endothelial barrier dysfunction via activation of the p38 MAPK/HSP27 pathway. Furthermore, EGCG selectively inhibits Ang II-induced p38 MAPK and HSP27 phosphorylation, resulting in suppression of endothelial stress fiber formation and hyperpermeability. Our observations explain the beneficial effects of physiological concentrations of green tea $(\leq 25 \mu \mathrm{mol} / \mathrm{L})$ in cardiovascular disease. Finally, our data suggest that the p38 MAPK/HSP27 pathway may be a novel target in the treatment of atherosclerotic cardiovascular disease.

\section{Acknowledgements}

This work was supported by grants from the National Natural Science Foundation of China (HL, 2006CB910306) and the 111 project (HL, B08007).

\section{Author contribution}

All of the authors guarantee the integrity of the study. Huihua LI designed the study concepts; Hui-hua LI, Yue-hong ZHENG, and Dan YANG designed the study; Hui-hua LI, Dan YANG, and Jing LIU researched the literature; Dan YANG and Yong ZENG acquired the data; Dan YANG, Jing LIU, and Cui TIAN analyzed and interpreted the data; Hui-hua LI, Dan YANG, and Jing LIU performed the statistical analyses; Jing LIU and Quan FANG prepared the manuscript; Dan YANG, Jing LIU, Quan FANG assisted in the manuscript preparation; Hui-hua LI, Yue-hong ZHENG, Quan FANG edited the manuscript; and Dan YANG and Jing LIU reviewed the manuscript. The final version was approved by all of the authors.

\section{References}

1 Brett J, Gerlach H, Nawroth P, Steinberg S, Godman G, Stern D. Tumor necrosis factor/cachectin increases permeability of endothelial cell monolayers by a mechanism involving regulatory g proteins. J Exp Med 1989; 169: 1977-91.

2 Bogatcheva NV, Dudek SM, Garcia JG, Verin AD. Mitogen-activated protein kinases in endothelial pathophysiology. J Investig Med 2003; 51: 341-52.

3 Wojciak-Stothard B, Entwistle A, Garg R, Ridley AJ. Regulation of TNF- 
alpha-induced reorganization of the actin cytoskeleton and cell-cell junctions by Rho, Rac, and Cdc42 in human endothelial cells. J Cell Physiol 1998; 176: 150-65.

4 Nakashima H, Suzuki H, Ohtsu H, Chao JY, Utsunomiya H, Frank GD, et al. Angiotensin II regulates vascular and endothelial dysfunction: recent topics of angiotensin II type-1 receptor signaling in the vasculature. Curr Vasc Pharmacol 2006; 4: 67-78.

5 Mehta PK, Griendling KK. Angiotensin II cell signaling: physiological and pathological effects in the cardiovascular system. Am J Physiol Cell Physiol 2007; 292: C82-97.

6 Suzuki Y, Ruiz-Ortega M, Lorenzo O, Ruperez M, Esteban V, Egido J. Inflammation and angiotensin II. Int J Biochem Cell Biol 2003; 35: 881-900.

7 Armstrong SC. Protein kinase activation and myocardial ischemia/ reperfusion injury. Cardiovasc Res 2004; 61: 427-36.

8 Xu SQ, Mahadev K, Wu X, Fuchsel L, Donnelly S, Scalia RG, et al. Adiponectin protects against angiotensin II or tumor necrosis factor alpha-induced endothelial cell monolayer hyperpermeability: role of cAMP/PKA signaling. Arterioscler Thromb Vasc Biol 2008; 28: 899905.

9 Hsu HH, Hoffmann S, Endlich N, Velic A, Schwab A, Weide T, et al. Mechanisms of angiotensin II signaling on cytoskeleton of podocytes. J Mol Med 2008; 86: 1379-94.

10 Crowley SD, Frey CW, Gould SK, Griffiths R, Ruiz P, Burchette JL, et al. Stimulation of lymphocyte responses by angiotensin II promotes kidney injury in hypertension. Am J Physiol Renal Physiol 2008; 295: F515-24.

11 Chen L, Zhang HY. Cancer preventive mechanisms of the green tea polyphenol (-)-epigallocatechin-3-gallate. Molecules 2007; 12: 94657.

12 Hofmann CS, Sonenshein GE. Green tea polyphenol epigallocatechin3-gallate induces apoptosis of proliferating vascular smooth muscle cells via activation of p53. Faseb J 2003; 17: 702-4.

13 Lorenz M, Wessler S, Follmann E, Michaelis W, Dusterhoft T, Baumann $\mathrm{G}$, et al. A constituent of green tea, epigallocatechin-3-gallate, activates endothelial nitric oxide synthase by a phosphatidylinositol3-OH-kinase-, cAMP-dependent protein kinase-, and Akt-dependent pathway and leads to endothelial-dependent vasorelaxation. J Biol Chem 2004; 279: 6190-5.

14 Widlansky ME, Hamburg NM, Anter E, Holbrook M, Kahn DF, Elliott JG, et al. Acute EGCG supplementation reverses endothelial dysfunction in patients with coronary artery disease. J Am Coll Nutr 2007; 26: 95-102.

15 Kim JA, Formoso G, Li Y, Potenza MA, Marasciulo FL, Montagnani M, et al. Epigallocatechin gallate, a green tea polyphenol, mediates NOdependent vasodilation using signaling pathways in vascular endothelium requiring reactive oxygen species and Fyn. J Biol Chem 2007; 282: 13736-45.

16 Yang D, Guo S, Zhang T, Li H. Hypothermia attenuates ischemia/ reperfusion-induced endothelial cell apoptosis via alterations in apoptotic pathways and JNK signaling. FEBS Lett 2009; 583: 2500-6.

17 Li HH, Kedar V, Zhang C, McDonough H, Arya R, Wang DZ, et al. Atrogin-1/muscle atrophy F-box inhibits calcineurin-dependent cardiac hypertrophy by participating in an SCF ubiquitin ligase complex. J Clin Invest 2004; 114: 1058-71.

18 Yang D, Xie P, Guo S, Li H. Induction of MAPK phosphatase-1 by hypothermia inhibits TNF-\{alpha\}-induced endothelial barrier dysfunction and apoptosis. Cardiovasc Res 2010; 85: 520-9.

19 Borbiev T, Birukova A, Liu F, Nurmukhambetova S, Gerthoffer WT,
Garcia JG, et al. P38 MAP kinase-dependent regulation of endothelial cell permeability. Am J Physiol Lung Cell Mol Physiol 2004; 287 : L911-8.

20 Garcia JG, Wang P, Schaphorst KL, Becker PM, Borbiev T, Liu F, et al. Critical involvement of p38 MAP kinase in pertussis toxin-induced cytoskeletal reorganization and lung permeability. FASEB J 2002; 16 : 1064-76.

21 Goldberg PL, MacNaughton DE, Clements RT, Minnear FL, Vincent PA. P38 MAPK activation by TGF-beta1 increases MLC phosphorylation and endothelial monolayer permeability. Am J Physiol Lung Cell Mol Physiol 2002; 282: L146-154.

22 Kevil CG, Oshima T, Alexander JS. The role of p38 MAP kinase in hydrogen peroxide mediated endothelial solute permeability. Endothelium 2001; 8: 107-16.

23 Kiemer AK, Weber NC, Furst R, Bildner N, Kulhanek-Heinze S, Vollmar AM. Inhibition of p38 MAPK activation via induction of MKP-1: Atrial natriuretic peptide reduces TNF-alpha-induced actin polymerization and endothelial permeability. Circ Res 2002; 90: 874-81.

24 Petrache I, Birukova A, Ramirez SI, Garcia JG, Verin AD. The role of the microtubules in tumor necrosis factor-alpha-induced endothelial cell permeability. Am J Respir Cell Mol Biol 2003; 28: 574-81.

25 Bogatcheva NV, Verin AD. The role of cytoskeleton in the regulation of vascular endothelial barrier function. Microvasc Res 2008; 76 : 202-7.

26 Obata T, Brown GE, Yaffe MB. MAP kinase pathways activated by stress: the p38 MAPK pathway. Crit Care Med 2000; 28: N67-77.

27 Kayyali US, Pennella CM, Trujillo C, Villa O, Gaestel M, Hassoun PM. Cytoskeletal changes in hypoxic pulmonary endothelial cells are dependent on MAPK-activated protein kinase MK2. J Biol Chem 2002; 277: 42596-602.

28 Wang Q, Doerschuk CM. The p38 mitogen-activated protein kinase mediates cytoskeletal remodeling in pulmonary microvascular endothelial cells upon intracellular adhesion molecule-1 ligation. J Immunol 2001; 166: 6877-84.

29 Guay J, Lambert H, Gingras-Breton G, Lavoie JN, Huot J, Landry J. Regulation of actin filament dynamics by p38 MAP kinase-mediated phosphorylation of heat shock protein 27. J Cell Sci 1997; 110: $357-$ 68.

30 Huot J, Houle F, Marceau F, Landry J. Oxidative stress-induced actin reorganization mediated by the p38 mitogen-activated protein kinase/ heat shock protein 27 pathway in vascular endothelial cells. Circ Res 1997; 80: 383-92.

31 Potenza MA, Marasciulo FL, Tarquinio M, Tiravanti E, Colantuono G, Federici A, et al. EGCG, a green tea polyphenol, improves endothelial function and insulin sensitivity, reduces blood pressure, and protects against myocardial I/R injury in SHR. Am J Physiol Endocrinol Metab 2007; 292: E1378-87.

32 Watson JL, Ansari S, Cameron H, Wang A, Akhtar M, McKay DM. Green tea polyphenol (-)-epigallocatechin-gallate blocks epithelial barrier dysfunction provoked by IFN-gamma but not by IL-4. Am J Physiol Gastrointest Liver Physiol 2004; 287: G954-61.

33 Chen C, Lu XH, Yan S, Chai H, Yao Q. HIV protease inhibitor ritonavir increases endothelial monolayer permeability. Biochem Biophys Res Commun 2005; 335: 874-82.

34 Natsume H, Adachi S, Takai S, Tokuda H, Matsushima-Nishiwaki $\mathrm{R}$, Minamitani $\mathrm{C}$, et al. (-)-Epigallocatechin gallate attenuates the induction of HSP27 stimulated by sphingosine 1-phosphate via suppression of phosphatidylinositol 3-kinase/Akt pathway in osteoblasts. Int J Mol Med 2009; 24: 197-203. 